\title{
33. Manipulation Planning for Knotting Linear Objects with One Hand
}

\author{
Tsunenori Kato, Hidefumi Wakamatsu, Akira Tsumaya, and Eiji Arai \\ Affiliation: Dept. of Manufacturing Science, Osaka Univ. \\ Em:\{kato,wakamatu, tsumaya, arai\}@mapse.eng.osaka-u.ac.jp
}

\begin{abstract}
A planning method for linear object manipulation, especially knotting is shown. At first, topological states of a linear object are described. Next, transitions between states are defined. Then, we can generate possible sequences of state transition, from which, we can choose an adequate path from the initial state to the objective state. Furthermore, a method to determine the grasping points is proposed. In the fourth a planning method is proposed. Finally, our system based on proposed methods is demonstrated
\end{abstract}

\section{INTRODUCTION}

In production sites, a lot of deformable linear objects like wires, codes, and cables are used widely; for data transmission, object transportation, fixing or packing of objects, and so on. However, systematic approach for realizing those manipulative tasks aimed at such deformable objects has not established yet. Because the physical property of them is diversity, it is very difficult to adopt the method for manipulating rigid objects.

Focusing on linear objects, especially, those applications are accompanied by knotting manipulation usually.

Wolter $e t$ al. have proposed the method to describe the deformation process of linear objects qualitatively (J.Wolter, 2001). Leaf has described deformed shape of fabric geometrically (G.A.V.Leaf, 1960). Morita at el. have proposed a system for knot planning from observation of human demonstrations (T.Morita, 2002). Matsuno at el. have realized a task of tying a cylinder with a rope by a dual manipulator system identifying the rigidity of the rope from visual information (T.Matsuno, 2001).

When we make a knot, we manipulate a linear object by several fingers of both hands for bending, twisting, and holding the linear object. The way to make several knots depends on human makeup or experience, so it is not unique. We can generate manipulation plans suitable for equipment and facilities with unlike physical makeup of human if processes for knotting a linear object can be modeled. Then, in this paper, we propose a method for automatic planning and execution of linear object manipulation which includes knotting.

At first, we propose qualitative crossing state of linear object in three dimensional space. Secondly, we propose a manipulation process of a linear object can be represented as a sequence of crossing state transition. Thirdly, it is shown that 
any manipulation process can be realized by one hand and a planning method for one-handed manipulation is proposed. Finally, we demonstrate a knotting experiment of an overhand knot performed by a vision-guided manipulator system to examine the usefulness of our approach.

\section{QUALITATIVE REPRESANTATION OF CROSSING STATES}

In this section, we define how to represent the state of a linear object qualitatively in order to generate manipulation planning.

At first, we define the state of a linear object as its projection on a plane. Then, on this projection plane, a curve may cross with itself. Note that how to cross of the $2 \mathrm{D}$ curve depends on the projection plane. Next, we number crossing points of the linear object along it. Then, the state of the linear object is represented as a set of $C_{i}$ $(i=1 \ldots, n)$ standing for crossing points, $E_{l}$ and $E_{r}$ standing for the left endpoint and the right endpoint respectively. Fig. 1 shows an example of a linear object. It has 5

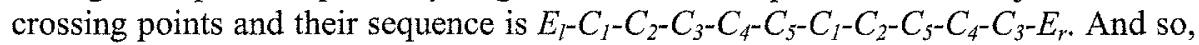
we can define the state of the object as a sequence of its crossing points. And then, at each crossing point, we define the upper part $C_{i}^{a}$ and the lower part $C_{i}^{l}$. Furthermore, we can distinguish two types of crossing; one of the two is the crossing that the upper part overlaps from the left side of the lower part to its right side and the other is opposite crossing. We define the former as the right hand helix crossing and the latter as the left hand helix crossing, and $\mathrm{C}^{+}{ }_{i}$ which stands for the right hand helix crossing, $C_{i}$ which stands for the left hand helix crossing. So, Fig.1 can be

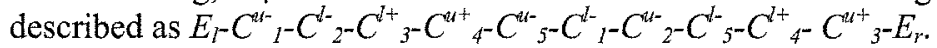

Consequently, we can represent the state of linear objects including knotted ones as finite crossing states qualitatively, regardless of its length, thickness, or other physical properties.

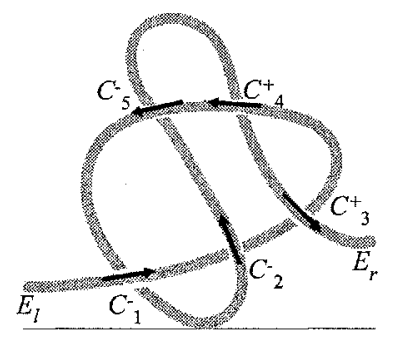

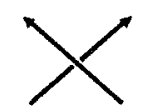

The left hand helix crossing

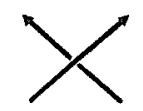

The right hand helix crossing

Fig.1 Example of knotted linear object Fig.2 The definition of two types of crossing

\section{DEFENITON OF OPERATIONS FOR STATE CHANGING}

In the previous section, we showed the states of linear objects can be represented by a sequence of crossing points. In this section, we consider how to transit the states of linear objects. In order to change the crossing state of a linear object, some operation must be performed on the object. Then, a state transition corresponds to an operation that changes the number of crossing points or rearranges their sequence. In this, to execute state transition of linear objects, four basic operations are prepared as shown in Fig.3. Operation type-I, II, and III are equivalent to Reidemeister move type-I, II, 
and III in the knot theory (C.C.Adams, 1994). By these operations, topology of the object state is not changed. But, type-IV operation in Fig.3 is regarded as an operation of changing topology. This operation is not included in Reidemeister, because in the knot theory, endpoints are not focused on. By operation type-I, II, IV, the number of crossing points is increased or decreased. Operation type-III does not change the number of crossing points but change their sequence. Let us define operations to increase crossing points as crossing operations $\mathrm{CO}_{\mathrm{I}}, \mathrm{CO}_{\mathrm{II}}$ and $\mathrm{C}_{\mathrm{IV}}$, operations to decrease them as uncrossing operations $\mathrm{UO}_{\mathrm{I}}, \mathrm{UO}_{\mathrm{II}}$, and $\mathrm{UO}_{\mathrm{IV}}$, and an operation keeping the number of them as an arranging operation $\mathrm{AO}_{\mathrm{III}}$.

The number of possible crossing states after a crossing operation can be much larger than those after an uncrossing operation. So, in this paper, a manipulation process can be represented as a sequence of uncrossing operations.

Fig.4 shows an example of a required manipulation. The initial state in Fig.4 (a)

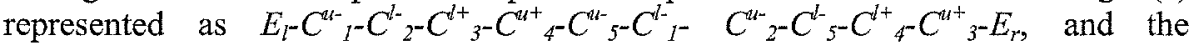
objective state in Fig.4 (b) represented as $E_{l}-E_{r}$. Fig.5 is a derived graph from the initial state to the objective state used uncrossing operations. In this graph, 14 crossing states and 32 state transitions are included. The example in Fig.5 shows unknotting processes. After deriving the sequence of uncrossing operations, by following it backward, knotting manipulation processes can be generated.

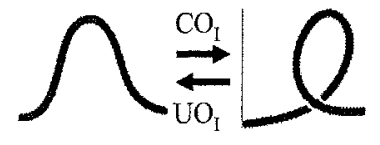

(a) type-I

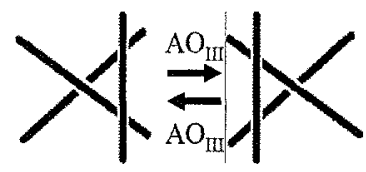

(c) type-III

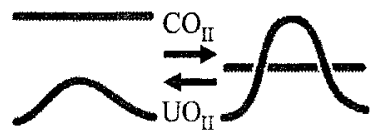

(b) type-II

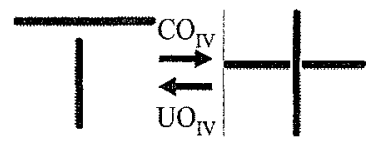

(d) type-IV

Fig.3 Basic operations

\section{PLANNING METHOD FOR ONE HANDED MANIPULATION}

In this section, a planning method for one-handed manipulation for linear objects is proposed. A crossing state graph in Fig.5 includes sequences which consist of typeIV operation alone. All manipulation tasks can be achieved by iteration of type-IV operations. Therefore, in this paper, we show the ability of one-handed manipulation using type-IV operation alone. We define a grasping point and the approach direction of a manipulator for type-IV operation as shown in Fig.6. Fig.6 (a) shows them for $\mathrm{CO}_{\mathrm{IV}}$ and Fig.6 (b) shows them for UO $\mathrm{IV}_{\mathrm{IV}}$. Fig.6 (a-2) and Fig.6 (b-2) shows the opposite crossing of the case illustrated in Fig.6 (a-1) and Fig.6 (b-1) respectively. We define the crossing shown in Fig.6 (a-1) and Fig (b-1) as the upend crossing and that of shown in Fig.6 (a-2) and Fig.6 (b-2) as the down-end crossing. It is found that the upper part is selected as the grasping point, in both crossing. Furthermore, manipulator can access the objects from the front side of the 
projection plane in both cases. So, type-IV operation can be achieved by one-handed manipulator approaching from the front side. It implies that, we can realize manipulation for linear objects by one hand without turning over the whole or partial of it when it is laid on a table.

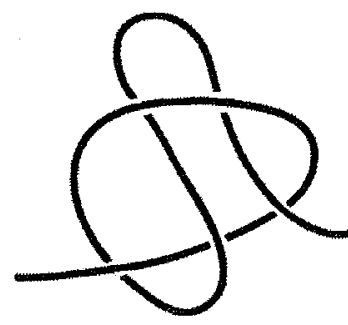

(a) initial state

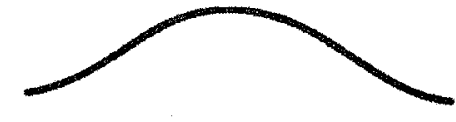

(b) objective state

Fig.4 Example of state transition

In uncrossing operations, the positions of points to be uncrossed are given. However, in crossing operations, the positions of points to be crossed are unknown. So, to execute crossing operations, they must be determined. In the objective state, a knotted object with $n$ crossing points has $2 n+1$ segments and $2 n$ upper/lower crossing points, that is, upper and lower points of crosses. Therefore, we divide the object into $2 n+1$ segments in the initial state. We define $D_{i}^{*}$ as a dividing point where subscript $*$ and subscript $i$ are equivalent to those of a crossing point created by crossing it.

In this section, we propose how to determine the length of each segment qualitatively. In this study, we assume left endpoint of linear object is fixed. Let us define some rules to determine length as follows. In these rules, $L$ and $S$ stand for long segment and short segment in comparison respectively.

Rule1. The segment between the fixed point and it adjoining point is defined as $L$.

Rule2. In the case of operating an up-end crossing, it is useful that the distance between a grasping point and the right endpoint is short. So, segments which exist in right side of the grasping point are defined as $\mathrm{S}$.

Rule3. In the case of operating a down-end crossing, segments existing between a grasping point and $C^{*} u$ are defined as $L$.

Rule4. After repeating those rules above, segments which are not determined are defined as $\mathrm{L}$.

Rule5. After repeating those rules above, segments which are determined more than two times are defined as their product.

By using these rules, we try determining length of each segment. Fig.7 shows an example of knotting manipulation. In this example, the initial state and the objective state are expressed respectively as follows:

$$
\begin{aligned}
& E_{l}-D_{1}^{l+}-D_{2}^{u+}-D_{3}^{l-}-D_{5}^{l+}-D_{1}^{u+}-D_{2}^{l+}-D_{5}^{u+}-D_{4}^{u-}-D_{3}^{l-}-E_{r} \\
& E_{l}-C_{1}^{l+}-C_{2}^{u+}-C_{3}^{l-}-C_{5}^{l+}-C_{1}^{u+}-C_{2}^{l+}-C_{5}^{u+}-C_{4}^{u-}-C_{3}^{l-}-E_{r}
\end{aligned}
$$

At first, the segment 1 in Fig. 8 becomes $L$ according to Rule1. And then, in the first operation in Fig.8, point $D_{l}^{{ }^{4+}}$ is grasped, moved and crossed on point $D_{l}^{l^{l+}}$ to realize 
this operation. In this time, the crossing operation to the up-end crossing is carried out. So, the segments $\mathbf{7}, \mathbf{8 , 9 , 1 0}$, and 11 becomes $S$ by Rule2. Next, in the second operation in Fig.8, point $D_{2}^{u+}$ is crossed on point $D_{2}^{l+}$, but in this case, $C_{1}{ }^{u+}$ exists in the right side of $D_{2}^{u+}$. So, the crossing operation to the down-end crossing is carried out. Thus, segments $\mathbf{3}, \mathbf{4}, \mathbf{5}$, and $\mathbf{6}$ become $L$ by Rule3, and in this time, the distance between $C_{l}^{u^{++}}$and $C_{l}^{l^{l+}}$ had been determined already, so the rest segment, i.e. segment 2 becomes $S$. Next, in the third operation and the fourth operation, the crossing operation to the up-end crossing is used. So, segment $9, \mathbf{1 0}$, and $\mathbf{1 1}$ are $S$ by Rule2. Finally, in the fifth operation, the crossing operation to the down-end crossing is used. So, segment $\mathbf{3 , 4 , 5}$ and $\mathbf{6}$ are $L$. Then, the length of each segment is determined as shown in Fig.8.

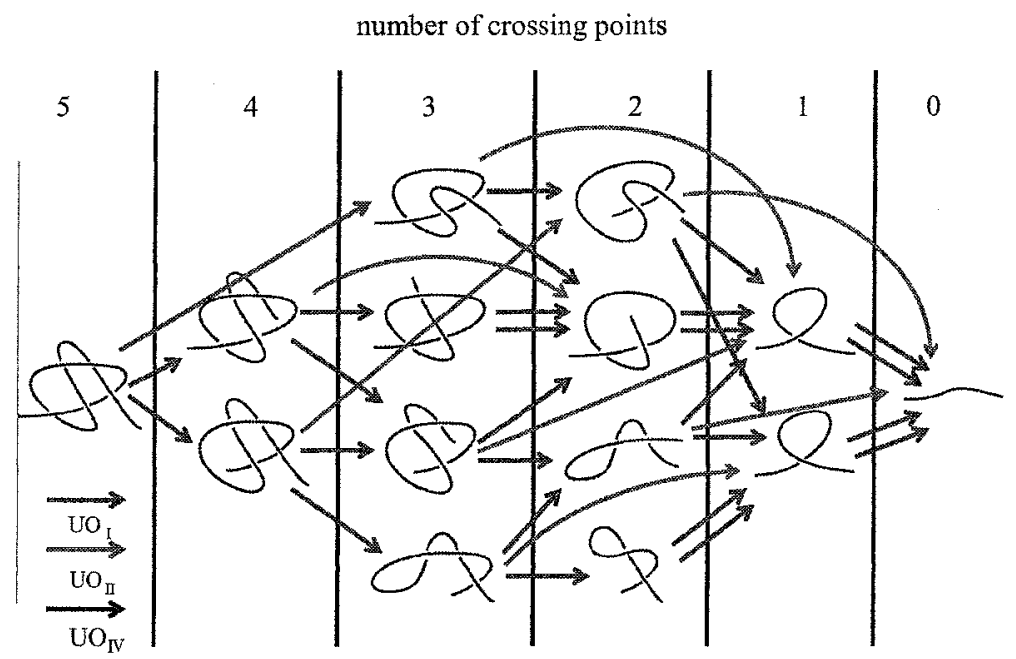

Fig.5 Result of manipulation process planning

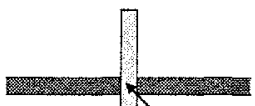

$\otimes$

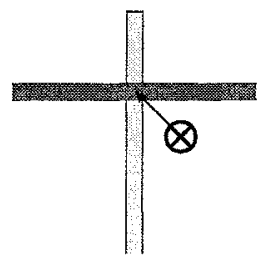

(a-1) up-end crossing (a-2) down-end crossing

(a) crossing operation
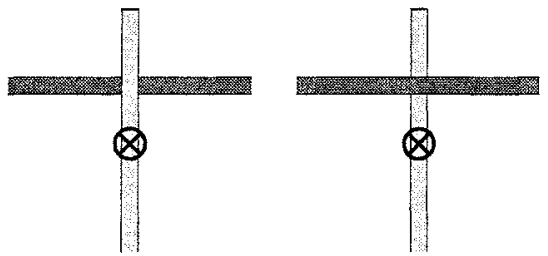

(b-1) up-end crossing (b-2) down-end crossing

(b) uncrossing operation

Fig.6 Grasping point for type-IV operation in crossing and uncrossing operation

Thus, if we substitute propriety numbers into $L$ and $S$, we can determine the real length of each segment. In addition to this example, to realize that manipulation, two points which make a crossing point should be moved so that they create right hand or left hand helix crossings properly. If the tangents at these points are given, we can generate possible trajectories of the manipulator to create crossing points. 


\section{CASE STUDY}

In this section, we demonstrate the validity of the method we have proposed in this paper. Fig. 9 shows concise view of our pilot system. It consists of PC for controlling a manipulator and image processing, a 6 DOF manipulator, and a CCD camera. We attempt to plan and carry out one-handed knotting manipulation with this system. $\mathrm{A}$ linear object, twist yarn, is laid on a table and its shape is captured by the camera fixed above the table. The table corresponds to a projection plane.

Fig. 10 shows a required manipulation. It corresponds to tying an overhand knot. The initial state and the objective state are shown in Fig.10 (a) and (b), respectively. They are represented as $E_{l}-E_{r}$ and $E_{l}-C^{l+}{ }_{1}-C^{u+}{ }_{2}-C^{l+}{ }_{3}-C^{u+}{ }_{1}-C^{l+}{ }_{2}-C^{u+}{ }_{3}-E_{r}$, respectively. Assumptions of this case study are as follows:

- The left endpoint of the object is fixed. A circle in Fig.10 represents the position of fixture.

- The manipulator releases the object whenever one crossing operation is finished.

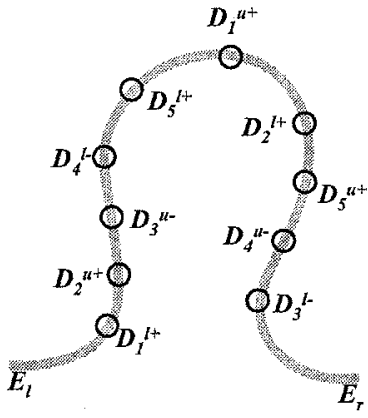

(a) initial state

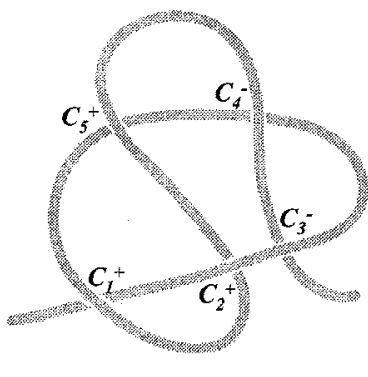

(b) objective state

Fig.7 Example of knotting manipulation

Then, one manipulation plan is generated as shown in Fig.11. This knotting manipulation consists of three $\mathrm{CO}_{\mathrm{rv}}$. The first and the third operations act on up-end crossing; the second operation is down-end crossing.

Next, this system can recognize the current crossing state of the object from a gray-scale image. The positions of crossing points in individual states are able to be identified by substituting suitable numbers into the $L$ and $S$ derived by the proposed.

About grasping points, in this paper, we define the upper point of each crossing point as a grasping point. And, direction of the axes can be calculated from the tangent at the grasping point. As appropriate moving distance of a manipulator for a state transition is unknown, the system checks whether the crossing state of the linear object is changed or not after moving the object. Thus, the manipulator can approach, grasp, move and release the object according to the generated qualitative plan. Fig. 12 shows the result of this manipulation.

Therefore, we think our proposed method we have proposed is effective for automatic planning and execution of linear object manipulation. 


\section{TOWARD DETAILED PLANNING}

We can plan manipulation for linear objects qualitatively by applying our proposed method. But it may be not enough to make a more complex knot like a bowknot, because a crossing operation to a down-end crossing in Fig. 6 is not certainty. So, in the case of carrying out a crossing operation to a down-end crossing, there is the necessity of preparations to make a down-end crossing certainly, for example pointing the right endpoint of the object to the grasping point in down-end crossing. If this situation comes true, quantitative analysis should be performed in order to check whether generated operations can be realized practically or not considering physical properties of a linear object. In quantitative analysis, the influence of the friction arisen by self-contact of the linear object is measurable, especially in knotting process. So, we had developed an analytical method to model the stable shape of a deformable linear object. Fig.13 shows the computed shape of an overhand knot, with/without the effect of friction.

Therefore, the manipulation strategy can be derived automatically by combining a qualitative planning proposed in this paper with the quantitative analysis.

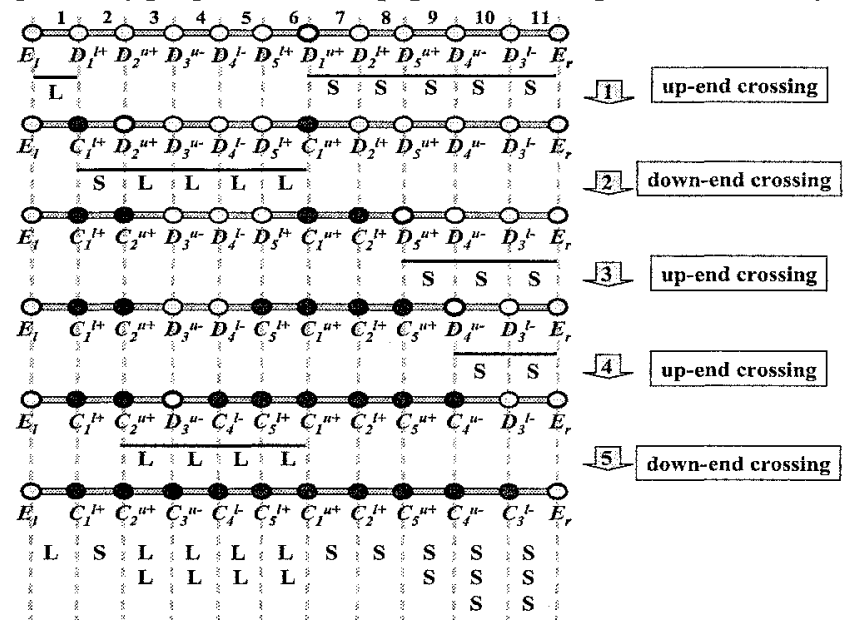

Fig. 8 Result of length of each segment

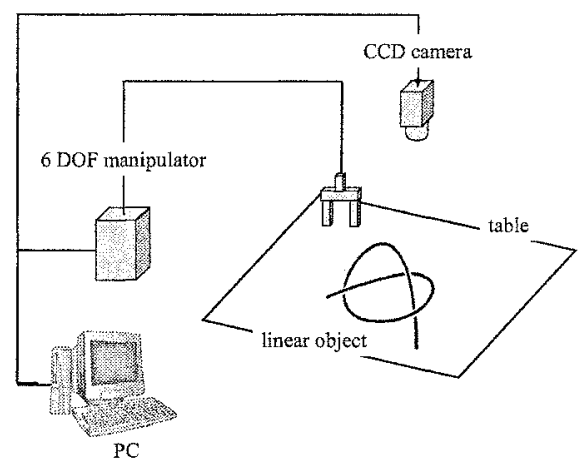

Fig.9 Overview of the experimental setup 

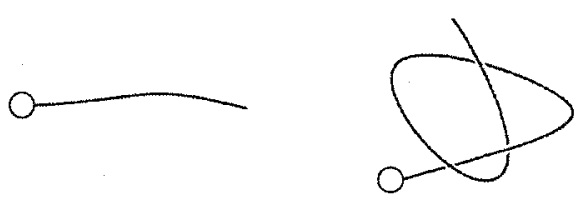
(a) initial state
(b) objective state

Fig.10 Required manipulation

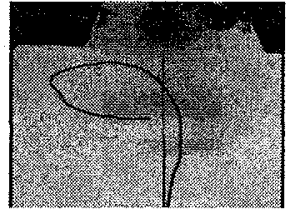

(a) initial state

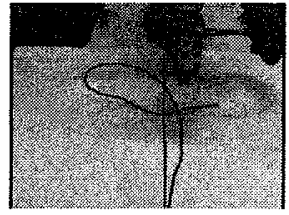

(b) after first operation

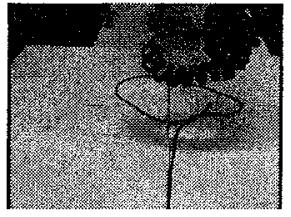

(c) after second operation

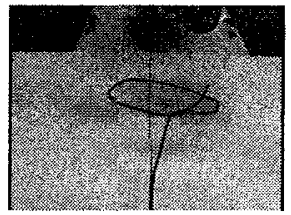

(d) after third operation

Fig.11 Generated manipulation plan
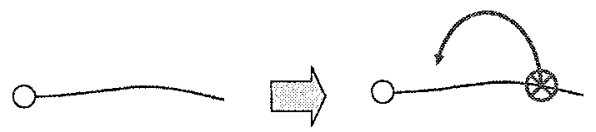

(a) first $\mathrm{CO}_{\mathrm{IV}}$
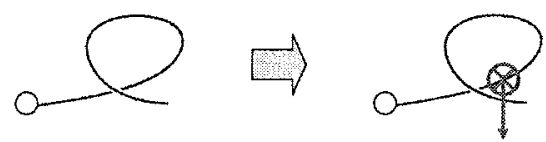

(b) second $\mathrm{CO}_{\mathrm{IV}}$

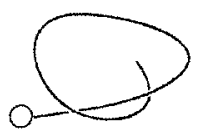

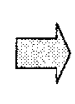
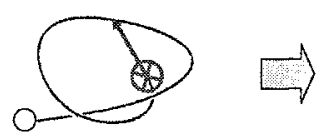

(c) third $\mathrm{CO}_{\mathrm{IV}}$
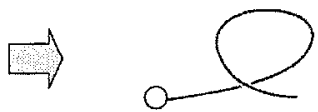<smiles>C1CCCC1</smiles><smiles>CC12CCCCCC(C1)O2</smiles><smiles>CC12CCC3(CC1)CC2C3</smiles>

Fig.12 Process of knotting manipulation 


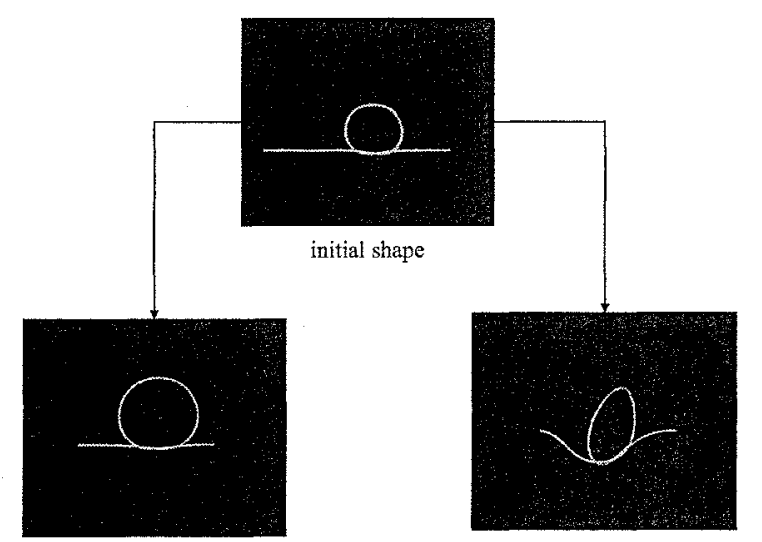

(a) shape of without influence of friction

(b) shape of with influence of friction

Fig.13 Quantitative analysis of influence of friction

\section{CONCLUSIONS}

In this paper, a planning method for linear object manipulation including knotting was proposed. Especially, it was shown that any knotting manipulation can be realized by one hand.

Firstly, a representation of topological states of a linear object was proposed. Its topological state can be represented as finite crossing states, and to execute transition between them, four basic operations were introduced. A state transition corresponds to a basic operation which changes the number of crossing points or permutated their sequence. So, giving the initial state and the objective state of a linear object, possible manipulation processes can be generated. Secondly, a planning method for one- handed manipulation was proposed because it was found that any manipulation is realized by one hand. Furthermore, a method for determination of grasping point was proposed by suggesting how to determine the length of each segment in order to realize derived manipulation processes. Finally, in order to demonstrate the effectiveness of our method, planning and execution of linear object manipulation by one hand was carried out.

\section{REFERENCES}

Adams, C.C (1994) The Knot Book: An Elementary Introduction to the Mathematical Theory of Knots, W.H.FREEMAN AND COMPANY

Leaf, G.A.V (1960) Models of the Plain-Knitted Loop, Journal of the Textile Institute, Vol.51, No.2, 135-171

Wolter, J. and Kroll, E (2001) Toward Assembly Sequence Planning with Flexible Parts, Proc, IEEE Int. Conf. on Advanced Intelligent Macaronis, 677-682.

Matsuno, T., Fukuda, T. and Arai, F (2001) Flexible Rope Manipulation by Dual Manipulator System Using Vision Sensor, Proc. of International Conference on Advanced Intelligent Mechatronics

Morita, T., Takamatsu, J., Ogawa, K., Kimura, H., Ikeuchi, K (2002) Knot Planning from Observation, Proc. of IEEE Int. Conf. Robotics and Automation 